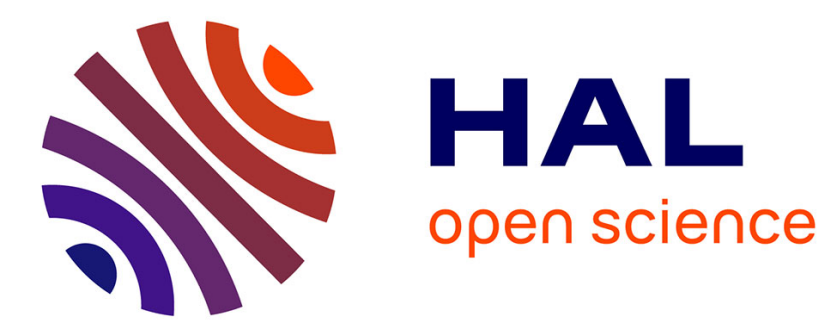

\title{
Cognitive fatigue: an impaired cortical inhibitory replenishment
}

Claude Touzet

\section{To cite this version:}

Claude Touzet. Cognitive fatigue: an impaired cortical inhibitory replenishment. Brain Injury, 2017, 8, pp.1 - 7. 10.1080/02699052.2017.1344300 . hal-01573271

\section{HAL Id: hal-01573271 \\ https://hal-amu.archives-ouvertes.fr/hal-01573271}

Submitted on 9 Aug 2017

HAL is a multi-disciplinary open access archive for the deposit and dissemination of scientific research documents, whether they are published or not. The documents may come from teaching and research institutions in France or abroad, or from public or private research centers.
L'archive ouverte pluridisciplinaire HAL, est destinée au dépôt et à la diffusion de documents scientifiques de niveau recherche, publiés ou non, émanant des établissements d'enseignement et de recherche français ou étrangers, des laboratoires publics ou privés. 


\title{
Cognitive fatigue: an impaired cortical inhibitory replenishment
}

\author{
C. Touzet - Aix Marseille Université
}

\begin{abstract}
Cognitive fatigue is an important symptom of many brain diseases, including Traumatic Brain Injury (TBI) and Multiple Sclerosis (MS). Cognitive fatigue shares many symptoms with chronic sleep deprivation, best described as cumulative excess wakefulness. If cognitive fatigue is caused by cumulative excess wakefulness, then - if the sleep duration is normal - the function of sleep itself must be impaired. Since sleep aims primarily to replenish the efficiency of cortical inhibitory synapses, it follows that TBI and MS must affect NonREM sleep (light and slow wave sleeps). Strategies providing a more efficient NonREM sleep could demonstrate some therapeutic action against cognitive fatigue.
\end{abstract}

Keywords: cognitive fatigue, partial sleep restriction, cumulative excess wakefulness, cortical inhibitory replenishment, SWS, NonREM sleep.

\section{Cognitive fatigue}

Fatigue is defined as "a subjective, unpleasant symptom which incorporates total body feelings ranging from tiredness to exhaustion creating an unrelenting overall condition which interferes with individuals' ability to function to their normal capacity" [1]. In the case of Traumatic Brain Injury (TBI), cognitive fatigue is reported by $43-73 \%$ of the patients, and is rated as the primary symptom by $7 \%$ of them, and one of the most troubling by $43 \%$ [2-6]. Stroke patients (cerebrovascular accident, CVA) [7-8] and Parkinson patients do also experience cognitive fatigue [9]. Fatigue is one of the most common symptoms reported in $70-90 \%$ of patients with Multiple Sclerosis (MS) [10-11], and 40\% of MS patients rate it to be their most troubling symptom. MS cognitive fatigue has received a lot of attention; we will refer to MS studies when TBI results are not available.

Fatigue is more troublesome and complex than one would think from the previous definition. Jacobs [12] described it in more vivid terms: "The fatigue that they experience defies description, going far beyond, and far deeper than anything a person with no brain injury would consider as profound exhaustion". Cognitive fatigue impacts negatively on the performance of activities such as walking, talking, eating, driving, shopping, cooking, going to school or working, including how often, for how long (i.e. endurance), and the all-important, how consistently (e.g. every day, once a week). Cognitive fatigue patients have to be careful of how many of these daily activities can be carried out sequentially during a given day, whereas people with no brain injuries don't have to.

The relation between (perceived) fatigue and objective cognitive performances was elusive until studies involving functional magnetic resonance imaging (fMRI), which allowed to objectify cognitive fatigue for MS patients [13-14]. Diffusion tensor imaging (DTI) revealed a relation between patterns of cerebral activation and increased self-reported fatigue [14] on the Fatigue Severity Scale (FSS, [15]). Another fMRI study by Kohl et al. involving TBI patients was also demonstrative of neural correlates of self-reported cognitive fatigue [16].

However, it is intriguing that numerous studies were not able to consistently correlate subjective and objective measures. It was even argued that behavioral performance was not the best measure of cognitive fatigue - then, more precise tests, with a much better score at evidencing a link between cognitive fatigue and decreased cognitive performance were developed [4]. For example, 
Johansson et al. [17] have showed that complex and demanding cognitive tasks (involving endurance, processing speed and attention) do reduce performance over time for individuals experiencing mental fatigue after brain injury.

Despite recent and more sensitive tests, the situation is still complex:

- A significant proportion of TBI (and MS) patients do not experience cognitive fatigue.

- Others have fatigue symptoms with no particular impact on behavioral performance as long as endurance is not required.

- Others (TBI) display an initially impaired cognition [18-23].

- Some TBI patients may be unable to improve their performance by training [24].

- Some TBI patients require more time to execute the task, but show the same level of performance [25].

Borgaro et al. [26] observed that the predominant items of the Barrow Neurological Institute (BNI) fatigue scale are (for TBI patients):

- "How difficult is it for me to stay awake during the day?"

- "How difficult is it for me to stay out of my bed during the day?"

- "How difficult is it for me to attend to something without becoming sleepy?"

- "How difficult is it for me to last the day without taking a nap?"

Fatigue appears to be strongly related to sleepiness. Does partial sleep deprivation show similarities with cognitive fatigue?

\section{Cumulative excess wakefulness}

Cognitive performance decreases as the day proceeds [27], and after the normal 16 hours of wake, performance decreases even more dramatically. Studies of chronic partial sleep deprivation have found clear evidence of performance impairment [28-30]. Laboratories-based studies of chronic partial sleep deprivation indicate that the effects are cumulative. Performance and alertness progressively worsen across days of sleep restriction. Sleep deprivation also induces effects such as the loss of endurance, loss of attention, loss of vigilance [31-33].

Van Dongen et al. [31] have studied the decrease of cognitive performance with a group of 48 healthy young adults (ages 21-38) submitted to partial or complete sleep deprivation. By comparing cumulative sleep debt versus cumulative excess wakefulness, they conclude that "sleep debt is perhaps best understood as resulting in additional wakefulness that has a neurobiological "cost" which accumulates over time". Figure 1 shows behavioral alertness (measured by Psychomotor Vigilance Task, PVT [34]) as a function of cumulative excess wakefulness [31]. The important parameter for cognitive impairment is therefore not how much sleep is missing, but how much wakefulness is in excess (to the normal 16 hours).

It is worth noting that extreme sleep deprivation subjects are prone to invasive sleepiness, falling 
asleep instantly, without warning - conditions that remind us of Jacobs' [12] vivid description of mental fatigue experienced by MS patients.

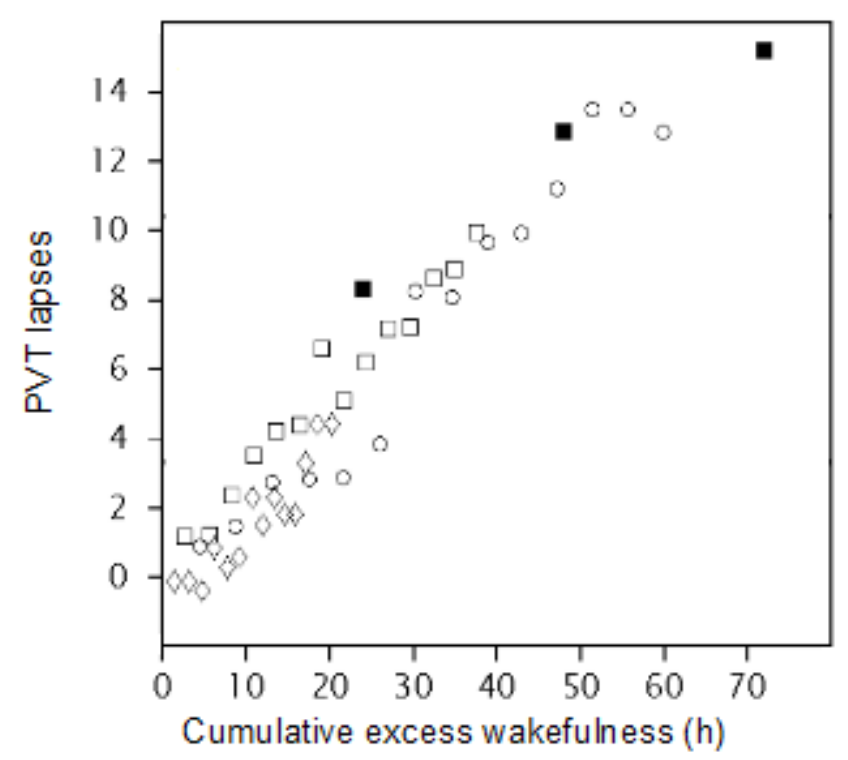

Figure 1. Four different sleep restrictions were used in this experiment: 8 h (i.e., total sleep restriction, black square), 6 hours (circle), 4 hours (light square) and 2 hours (diamond) per night. The first black square points the cognitive performance after a normal 16 hours of wake, plus an additional 24 hours of wakefulness (without any sleep). Cumulative excess wakefulness starts after a normal $16 \mathrm{~h}$ of wake. The next black square is obtained after a second day of total sleep deprivation $(24+24 \mathrm{~h})$. This panel illustrates the monotonic, near-proportional relation between cumulative excess wakefulness and neurobehavioral performance impairment irrespective of daily sleep ration (from [31]).

\section{Neural basis of sleep}

The neurobiological "cost" of Van Dongen et al. has been hypothesized as being the erosion of the cortical inhibitory synapse efficiency [35]. The idea is that hebbian learning guarantees that efficient inhibitory synapses lose their efficiency during the day - just because they are efficient at avoiding the activation of the targeted neurons. Since hebbian learning is the only known mechanism of synapse modification [36], it follows that to replenish the inhibitory synapses' efficiency [37-38], source and targeted neurons must be activated together [39]. A traveling wave of depolarization [40] is exactly what is needed to ensure the strengthening of local inhibitory synapses. This describes exactly the "slow-wave sleep" (SWS). Figure 2 illustrates this neural regulatory mechanism [35].

This sleep hypothesis states that the purpose of paradoxical sleep (Random Eye Movements sleep - REM), which follows each session of SWS, is to counteract the eroding effect of SWS on the excitatory long distance connections which code previous day events. Indeed REM activity resembles that of the awake state, except that it is played much faster and not in the same order [41]. This hypothesis explains the synergy between NonREM (Light Sleep and SWS) and REM sleeps which act together in order to guarantee that when the subject awakes again, his inhibitory synaptic efficiency is restored and his (excitatory) long distance connection adjustments have been preserved. 


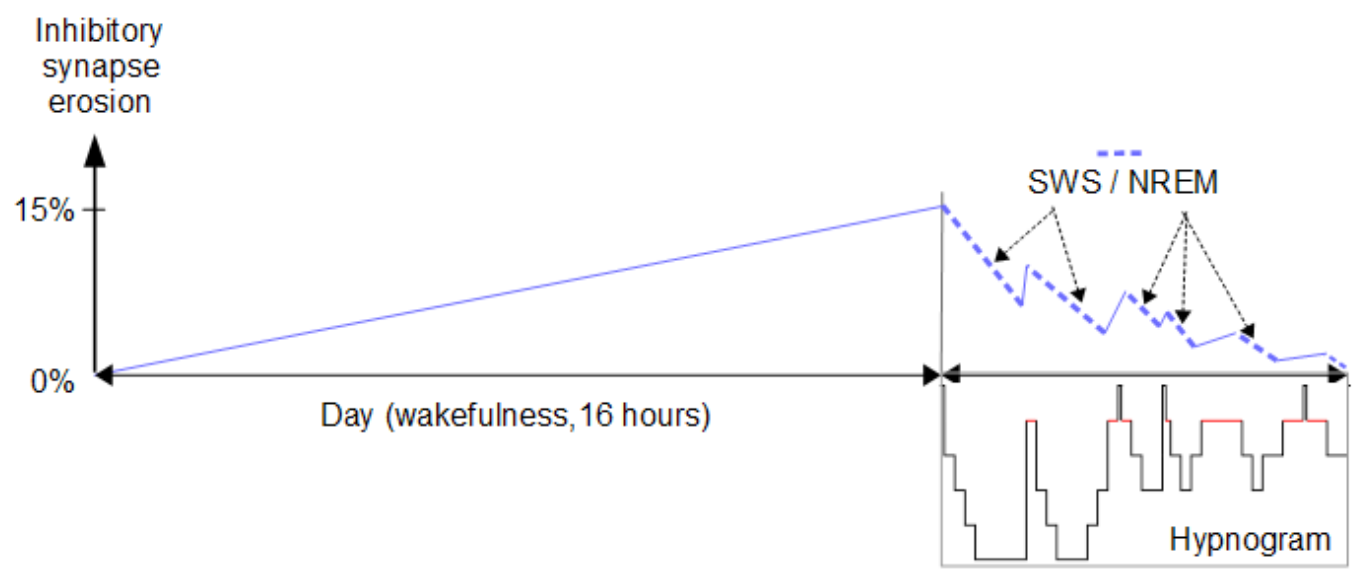

Figure 2. During the 16 hours of wakefulness, inhibition efficiency is reduced by an amount of $15 \%$. During the 8 hours of night, NonREM sleep reduces the inhibition erosion so that at wake time, there is no erosion left. REM sleep intervenes after each NonREM period in order to preserve the long distance excitatory connection updates (memory of previous day). REM sleep has a deleterious effect on inhibitory connection efficiency (modified from [35]).

The numbers shown Table 1 [35] determine both the duration and the neural recruitment of each sleep period. REM and SWS recruitment numbers are similar (100\%), when light sleep (LS) recruits only half (50\%) and wakefulness recruits 5 times less $(20 \%)$ neurons.

Table 1. Duration and percentage of neurons recruited over a 24 hours day (corrected from [35]).

\begin{tabular}{|l|c|c|c|c|}
\hline Effect on synapses & \multicolumn{2}{|c|}{$\begin{array}{c}\text { Reinforcement of excitatory and decrease } \\
\text { of inhibitory connections }\end{array}$} & \multicolumn{2}{c|}{$\begin{array}{c}\text { Reinforcement of inhibitory and decrease } \\
\text { of excitatory connections (NonREM) }\end{array}$} \\
\hline Period of the day & Wakefulness & REM & LS (Light Sleep) & SWS \\
\hline Repartition / day & 16 hours & $1 \mathrm{~h} 30$ & $3 \mathrm{~h} 30$ & $3 \mathrm{~h}$ \\
\hline \multirow{2}{*}{$\begin{array}{c}\text { \% recruitment } \\
\text { 20\% (5 times less } \\
\text { than REM) }\end{array}$} & $100 \%$ & $50 \%$ & $\begin{array}{c}100 \% \text { (maximal } \\
\text { as in REM) }\end{array}$ \\
\hline $\begin{array}{c}\text { Hours equivalent } \\
100 \% \text { recruiting }\end{array}$ & $3 \mathrm{~h} 15$ & $1 \mathrm{~h} 30$ & $1 \mathrm{~h} 45$ & $3 \mathrm{~h}$ \\
\cline { 2 - 5 } & \multicolumn{2}{|c|}{$4 \mathrm{~h} 45$} & \multicolumn{2}{c|}{$4 \mathrm{~h} 45$} \\
\hline
\end{tabular}

From the description made of the neural basis of sleep, it follows that a slight reduction of NonREM sleep percentage of recruitment, and/or its duration, is a sufficient condition to induce a chronic excess of wakefulness.

\section{Hypothesis}

As described, cumulative excess wakefulness impacts cognitive performance, attention, vigilance, endurance, which are all symptoms of cognitive fatigue. Could it be that patients with cognitive fatigue are in fact patients with a subnormal sleep recovery efficiency which maintains them in a chronic cumulative excess wakefulness condition? 


\section{Neural injuries in TBI and MS}

Embedded by thalamo-cortical loops, spindles induce LS [42], the precursor of SWS. Any deficiency of the thalamo-cortical loops will affect SWS:

- TBI are mainly acceleration/deceleration of the enclosed brain where the various structures (defined because of their homogeneity) will experience differential accelerations which generate cuttings at their frontiers. Due to the size of the cortex and the thalamus (respectively $82 \%$ and $12 \%$ of the central nervous system), connections between both structures (thalamo-cortical loops) are certainly among those that are the most impacted.

- As for MS, a default in axon conductance may be particularly harmful for the thalamocortical neurons' synchronization [43], therefore reducing the quality of the slow waves (a lesser amount of synchronization).

\section{Reduced NonREM sleep for patients with cognitive fatigue}

Kaynak et al. [44] have conducted polysomnographic studies in order to compare MS patients (with fatigue and no fatigue) to healthy persons. Their data show that MS patients do exhibit about $11 \%$ less duration of NonREM sleep (and also 11\% less REM sleep) than normal. We will use this value as representative of the NonREM sleep impairment. A normal 8 hours night is therefore not able to compensate for the whole day use, but only for $89 \%$. The problem is that normally, during the 16 hours wake time, there is more cognitive use than the putative $89 \%$ that can be replenished. Therefore, at wake the following morning, the cognitive performance level is already impacted, and things will only get worse the following days as shown on figure 3.

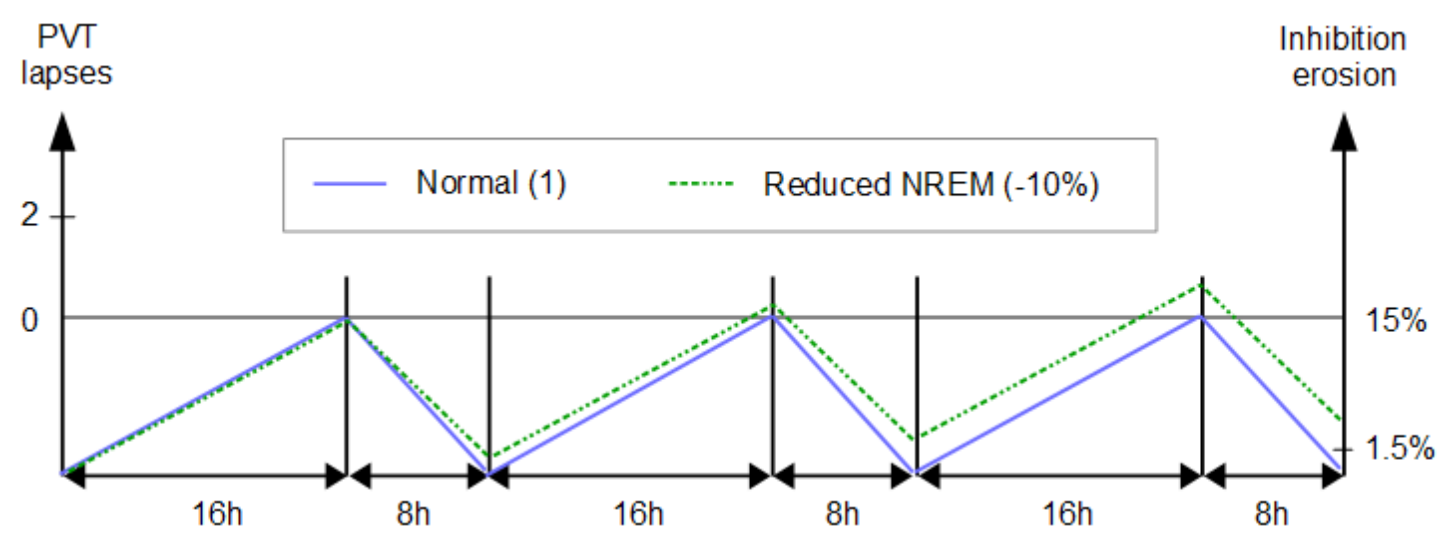

Figure 3. Cumulative erosion of the cortical inhibition efficiency and the associated neurobehavioral impairment (PVT lapses) for normal (1) and reduced NonREM sleep. The NonREM sleep efficiency of the impairment condition is "almost" normal at $89 \%$, however cognitive impairment quickly builds-up. After three days, the level of inhibitory erosion at wake is similar to the level of erosion after 4 hours of awakening - which means that at bedtime the cognition will be equivalent to that of a $4 \mathrm{~h}$ cumulative excess wakefulness. Inhibition erosion per day (of $16 \mathrm{~h}$ ) is $15 \%$. For PVT lapses axis, the performance level after a normal $16 \mathrm{~h}$ day (wakefulness) is set to " 0 ".

Theoretically, after a few days, the amount of cumulative excess wakefulness should be so huge ( $1.5 \mathrm{~h}$ x number-of-days) that no cognition could be displayed. Therefore, there must be a limiting mechanism that stops the cognition's loss of performance (before it goes too bad). A logical 
candidate would be sleep, with the patient's suggestibility to sleep (sleepiness / fatigue) building up as quickly as cumulative excess wakefulness.

Indeed a decreased efficiency of cortical inhibition favors the emergence of sleep since a lesser cortical inhibition favors thalamo-cortical synchronization [45-46]. Cognition would be impaired at wake, and get worse as time goes on until intruding sleep steps in. The interaction between sleepiness and wakefulness will settle around a certain equilibrium that could be considered as resulting from a regulation process (homeostasis).

\section{Equilibrium point}

What would be the equilibrium point between cumulative excess wakefulness and intruding sleep induction? It is certainly dependent on the patient, and therefore will manifest as patients having little or strong cognitive performance impairment at wake. However, it is worth noting that this homeostasis around an equilibrium will be reached independently of the number of damaged thalamo-cortical loops. Light or severe TBI patients, as well as mild or severe MS patients, are subject to this homeostasis, as shown on Figure 4. Less severe conditions will only take longer to settle to chronic fatigue. Healthy persons are also subject to this homeostasis but do not normally experience intruding sleep during a normal day.

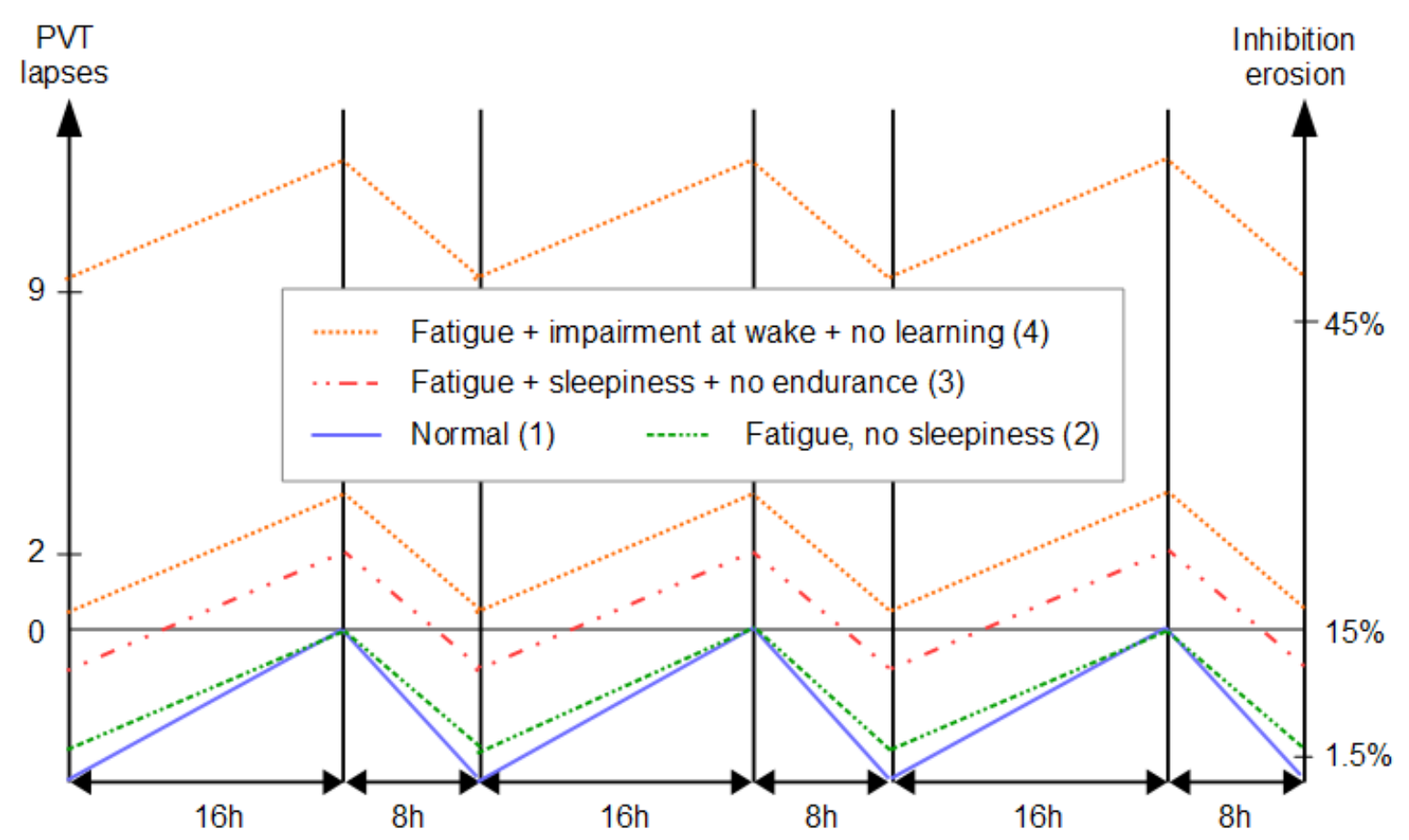

Figure 4. In the chronic state, when a point of equilibrium has been reached, the daily spending of "cognition" counterbalances exactly the amount that can be restored. Therefore, less cognition spending is allowed. Depending on the point of equilibrium, the patient may feel fatigue by the end of the day (2), or sooner (3), even at wake (4). Cognition will also be impacted in various manners, from almost normal cognition (2) to mildly impaired cognition and poor endurance (3), to strongly impaired cognition already at wake and no learning ability (4). An inhibition erosion of $45 \%$ is equivalent to $32 \mathrm{~h}$ of excess wakefulness ( $16 \mathrm{~h}$ normal day $+32 \mathrm{~h}$ ). 


\section{Fatigued vs non-fatigued patients}

What separates fatigued from non-fatigued patients is the (high vs low) level of inhibition erosion of their personal equilibrium point. After comparing fatigued vs non-fatigued MS patients, magnetic resonance spectroscopy (MRS) studies have shown significant reductions in $\mathrm{N}$-acetylaspartate/creatine $(\mathrm{NAA} / \mathrm{Cr}$ ) ratios in multiple brain regions among fatigued $\mathrm{MS}$ patients, suggesting axonal loss as a contributing factor [47]. Following our hypothesis, significant axonal loss is to be interpreted as a more severe impairment of NonREM sleep efficiency (more than 11\%), which results in a lesser amount of "cognition" available for the day.

\section{Discussion}

Our hypothesis is that cognitive fatigue results from cumulative excess wakefulness induced by an impaired NonREM Sleep. It agrees with the 5 bullets points describing cognitive fatigue know-how in TBI and MS patients (cf. section 1):

1. A significant proportion of TBI (and MS) patients do not experience cognitive fatigue. Very low homeostasis levels do not allow cumulative excess wakefulness to build-up (Fig. 4, case 2). Also, unlike performance measures, sleepiness ratings appeared to show adaptation to chronic partial sleep deprivation. Van Dongen et al. [31] report that "these findings for subjective sleepiness suggest that once sleep restriction is chronic, subjects either cannot reliably introspect with regard to their actual sleepiness levels, or as long as they are receiving at least approximately $4 \mathrm{~h}$ of sleep nightly they do not experience a sense of sleepiness anywhere near the levels found for total sleep deprivation." This inability to judge one's own sleepiness may account for a number of MS and TBI patients that do not report cognitive fatigue.

2. Others have fatigue symptoms with no particular impact on behavioral performance as long as endurance is not required. These could be patients with a homeostasis that allows only a very limited level of inhibitory synapse erosion before intruding sleep steps in (Fig. 4, case 3). Their equilibrium point is close to a normal cognitive performance, which allows them a certain amount of "normal" cognitive performance, but they quickly show degraded performance (no endurance).

3. Others display an initially impaired cognition. Patients having an equilibrium point that is associated with more than 16 hours of wake time (e.g. $16+32$ hours) will show initially strong impairment (Fig. 4, case 4).

4. Some patients may be unable to improve their performance by training. NonREM sleep is implicated in cognitive performance recovery, and REM sleep is implicated in avoiding the loss of the associations learned during the day (i.e., memory). If REM sleep regulation is impacted by TBI or MS, then it may be that there is a less efficient REM sleep which prohibits the consolidation of learned knowledge. NonREM and REM sleeps are sustained by different neural circuits, e.g. cortico-hippocampal loops are necessary for REM sleep (among others circuits).

5. Some patients require more time to execute the task, but show the same level of performance. Cognitive speed depends on the availability of long distance excitatory connections that allow the associations between various cortical maps, each one representing a specific dimension of the data [48]. If some of these connections are destroyed then the number of neural relays between two given maps increases, which is objectified by a speed reduction of cognitive processing. In the TBI case, the probability of cutting depends on the length of the connection. In the MS case, the probability of axon degradation depends on the number of neurons involved in the cognitive 
task. In both cases, brain injuries impose additional neurons, i.e., additional time to process the data.

Our hypothesis targets sleep efficiency, not sleep disturbance. Therefore, it accommodates the absence of a clear relation between sleep disturbance and cognitive fatigue. For example, sleep disturbance is frequent among TBI patients (50-73\%) [49-50], and is responsible for fatigue. However, a number of patients with fatigue do not exhibit sleep disturbance and not all patients with sleep disturbance are experiencing fatigue. Clinchot et al.[51] estimate that $50 \%$ of TBI patients show sleep disturbance, $63 \%$ are experiencing fatigue and among the $50 \%$ with sleep disturbance, $80 \%$ do experience fatigue.

Our hypothesis also agrees with the fact that fatigue does not appear more frequent between mild and severe cases of TBI [52], and that fatigue affects gender in the same proportion, whereas in the healthy population, fatigue is predominant in females (sex-ratio : 3/2) [52-53]. Our hypothesis also agrees with the fact that fatigue is not correlated to age, severity of the cognitive impairment or delay after injury [26,52].

As stated by Bradley [53], treatment of (MS-related) fatigue is challenging. DeMarchi et al.'s [54] literature survey of awakening agents (psycho-stimulants, antidepressants, anticonvulsants, etc.) shows that there is no clear proof of efficacy, and sometimes gives contradictory results. This is in accordance with our hypothesis asserting that sleepiness should not be considered as the problem, but as part of the solution instead.

\section{Conclusion and directions for further research}

Our hypothesis states that cognitive fatigue for TBI and MS patients is the result of impaired SWS, which is not able in a "normal" 8 hour night to replenish the cortical inhibitory synapse efficiency completely. The patient's fatigue is then similar to the cognitive fatigue experienced during chronic partial sleep deprivation by healthy people. In order to recover, a sleep debt must be slept. It follows that patient's fatigue may be alleviate by improving NonREM sleep efficiency and/or duration.

In order to validate this hypothesis, future studies should evaluate the benefits of an improved SWS efficiency on the cognitive fatigue symptom. Various intervention tools have already proven effective at improving SWS and cognition performance of healthy subjects. Similar studies should be conducted with fatigued patients. For example, for healthy subjects, a twenty minute t-DCS stimulation $(0.75 \mathrm{~Hz})$ during the first SWS period improves performance in memory recall by up to $5 \%$ the next morning, and also speed [55]. Building on these results, several commercial products have already reached customers. Sounds [56], such as binaural sounds are another tool able to strengthen the slow waves [57], and commercial products based on this technology are also available. Another possibility is provided by cutaneous temperature manipulation [58]. A $0.4^{\circ} \mathrm{C}$ skin temperature increase almost doubles the proportion of nocturnal slow wave sleep in elderly subjects.

These intervening studies should not conceal the fact that simple interventions may be of interest. For example, napping is also to be nurtured, since it allows a partial replenishment of cortical inhibition at midday. An extension of sleep duration is also of interest, as should be the drugs that potentiate NonREM sleep. A clear adhesion to the chronobiology is to be favored since the "best" sleep takes place in the early evening [59]. Sleep disturbance, even if it is not the primary cause of an impaired SWS efficiency, shouldn't be overlooked since it may also affect negatively sleep 
quantity and/or quality [60].

Last but not least, since the problem is caused by the cortical erosion of inhibition, a reduction of the inhibition's plasticity may help. A potential lead in this direction is offered by Morvan's syndrome, which symptoms include total insomnia without cognitive impact [61].

\section{Conflict of interest statement}

The author declares no conflict of interest associated with this publication.

\section{Acknowledgment}

We greatly thank Elise Millet for proofreading the manuscript.

\section{References}

1. Aaronson LS, Teel CS, Cassmeyer V, Neuberger GB, Pallikkathayil L, Pierce J, Press AN, Williams PD, Wingate A. Defining and measuring fatigue. Image-Journal Nurs Scholarchip 1999;31:45-50.

2. LaChapelle DL, Finlayson MA. An evaluation of subjective and objective measures of fatigue in patients with brain injury and healthy controls. Brain Inj 1998;12:649-59.

3. Olver JH, Ponsford JL, Curran CA. Outcome following traumatic brain injury: a comparison between 2 and 5 years after injury. Brain Inj 1996; 10:841-8.

4. Van der Naalt J, Van Zomeren AH, Sluiter WJ, Minderhoud JM. One year outcome in mild to moderate head injury: the predictive value of acute injury characteristics related to complaints and return to work. J Neurol Neurosurg Psychiatry 1999;66:207-13.

5. Van Zomeren AH, Van den Burg W. Residual complaints of patients two years after severe head injury. J Neurol Neurosurg Psychiatry 1985;48: 21-8.

6. Belmont A, Agar N, Hugeron C, Gallais B, Azouvi P. Fatigue and traumatic brain injury. Ann Readapt Med Phys 2006;49, 283-288.10.1016/j.annrmp.2006.04.017.

7. Glader EL, Stegmayr B, Asplund K. Poststroke fatigue: a 2-year follow-up study of stroke patients in Sweden. Stroke 2002;33:1327-33.

8. Ingles JL, Eskes GA, Phillips SJ. Fatigue after stroke. Arch Phys Med Rehabil 1999;80:1738.

9. Lou JS, Kearns G, Oken B, Sexton G, Nutt J. Exacerbated physical fatigue and mental fatigue in Parkinson's disease. Mov Disord 2001;16:190-6.

10. Fisk JD, Pontefract A, Ritvo PG, Archibald CJ, Murray TJ. The impact of fatigue on patients with multiple sclerosis. Can J Neurol Sci 1994;21: 9-14. PubMed: 8180914.

11. Krupp LB, Alvarez LA, LaRocca NG, Scheinberg LC. Fatigue in multiple sclerosis. Arch Neurol 1988;45: 435-437. doi:10.1001/archneur. 1988.00520280085020.

12. Jacobs L. (2011). Mild Brain Injury: Implications for Independence. Retrieved July, 16, 2016. Available from http://lanojake.wordpress.com/2011/12/16/mild-brain-injuryimplications-for-independence/

13. DeLuca J, Genova HM, Hillary FG, Wylie G. Neural correlates of cognitive fatigue in 
Publication acceptée par Brain Injury (identifiant TBIN-2016-0272) à paraître en 2017

multiple sclerosis using functional MRI. J Neurol Sci 2008;270: 28-39. doi:10.1016/j.jns.2008.01.018.

14. Genova HM, Rajagopalan V, DeLuca J, Das A, Binder A, Arjunan A, Chiaravalloti N, Wylie G. Examination of cognitive fatigue in multiple sclerosis using functional magnetic resonance imaging and diffusion tensor imaging. PloS one 2013;8(11), e 78811.

15. Krupp LB, LaRocca NG, Muir-Nash J, Steinberg AD. The fatigue severity scale. Application to patients with multiple sclerosis and systemic lupus erythematosus. Arch Neurol 1989;46:1121-3.

16. Kohl AD, Wylie GR, Genova HM, Hillary FG, Deluca J. The neural correlates of cognitive fatigue in traumatic brain injury using functional MRI. Brain Inj 2009;23(5): 420-432.

17. Johansson B, Rönnbäck L. Novel computer tests for identification of mental fatigue after traumatic brain injury. NeuroRehabilitation 2015;36(2), 195-202.

18. Azouvi P, Couillet J, Leclercq M, Martin Y, Asloun S, Rousseaux M. Divided attention and mental effort after severe traumatic brain injury. Neuropsychologia 2004;42, 1260-1268.

19. Belmont A, Agar N, Azouvi P. Subjective fatigue, mental effort, and attention deficits after severe traumatic brain injury. Neurorehabil Neural Repair 2009;23(9), 939-944.

20. Johansson B, Berglund P, Rönnbäck L. Mental fatigue and impaired information processing after mild and moderate traumatic brain injury. Brain Inj 2009;23(13-14), 1027-1040.

21. Park NW, Moscovich M, Robertson IH. Divided attention impairments after traumatic brain injury. Neuropsychologia 1999;37(10), 1119-1133.

22. Ponsford J, Cameron P, Fitzgerald M, Grant M, Mikocka-Walus A. Long-term outcomes after uncomplicated mild traumatic brain injury: A comparison with trauma controls. J Neurotrauma 2011;28, 937-946.

23. Ziino C, Ponsford J. Selective attention deficits and subjective fatigue following traumatic brain injury. Neuropsychology 2006;20, 383-390.

24. Ashman TA, Cantor JB, Gordon WA, Spielman L, Egan M, Ginsberg A, Engmann C, Dijkers M, Flanagan S. Objective measurement of fatigue following traumatic brain injury. J Head Trauma Rehabil 2008;23(1), 33-40.

25. Ozen LJ, Fernandes MA. Slowing down after a mild traumatic brain injury: A strategy to improve cognitive task performance? Arch Clin Neuropsycho 2012;27(1), 85-100.

26. Borgaro SR, Gierok S, Caples H, Kwasnica C. Fatigue after brain injury: initial reliability study of the BNI Fatigue Scale. Brain Inj 2004;18:685-90.

27. Blatter K, Cajochen C. Circadian rhythms in cognitive performance: methodological constraints, protocols, theoretical underpinnings. Physiology \& behavior 2007;90(2), 196208.

28. Dinges DF, Pack F, Williams K, Gillen KA, Powell JW, Ott GE, Aptowicz C, Pack AI. Cumulative sleepiness, mood disturbance, and psychomotor vigilance performance decrements during a week of sleep restricted to 4-5 hours per night. Sleep 1997; 20:267-277.

29. Tilley AJ, Wilkinson RT. The effects of a restricted sleep regime on the composition of sleep and on performance. Psychophysiology 1984; 21:406-412.

30. Drake CL, Roehrs TA, Burduvali E, Bonahoom A, Rosekind M, Roth T. Effects of rapid 
Publication acceptée par Brain Injury (identifiant TBIN-2016-0272) à paraître en 2017

versus slow accumulation of eight hours of sleep loss. Psychophysiology. 2001; 38:979-987.

31. Van Dongen HPA, Maislin G, Mullington JM, Dinges DF. The cumulative cost of additional wakefulness: dose-response effects on neurobehavioral functions and sleep physiology from chronic sleep restriction and total sleep deprivation. Sleep 2003;2:117-26.

32. Belenky G, Wesensten NJ, Thorne DR, Thomas ML, Sing HC, Redmond DP, Russo MB, Balkin TJ. Patterns of performance degradation and restoration during sleep restriction and subsequent recovery: a sleep-dose response study. J Sleep Res 2003; 12:1-12.

33. Alhola P, Polo-Kantola P. Sleep deprivation: Impact on cognitive performance. Neuropsychiatric disease and treatment 2007;3(5), 553.

34. Dorrian J, Rogers NL, Dinges DF. Psychomotor vigilance performance: A neurocognitive assay sensitive to sleep loss. In: Kushida C (Ed), Sleep Deprivation: Clinical Issues, Pharmacology and Sleep Loss Effects. Marcel Dekker, Inc., NY, pp. 39-70, 2005.

35. Touzet C. Sleep: the hebbian reinforcement of the local inhibitory synapses. Med. Hypotheses 2015;85:359-364.

36. Lamsa K, Heeroma JH, Kullmann DM. Hebbian LTP in feed-forward inhibitory interneurons and the temporal fidelity of input discrimination. Nat Neurosci. $2005 \mathrm{Jul} ; 8(7)$ : 916-24.

37. Scelfo B, Sacchetti B, Strata P. Learning-related long-term potentiation of inhibitory synapses in the cerebellar cortex, PNAS 2008;105(2):769-774. DOI:10.1073/ pnas.0706342105

38. Kullmann DM, Moreau AW, Bakiri Y, Nicholson E. Plasticity of Inhibition. Neuron 2012;75(6), 951-962. doi:10.1016/j.neuron.2012.07.030.

39. Woodin MA, Ganguly K, Poo MM. Coincident pre- and post-synaptic activity modifies GABAergic synapses by post-synaptic changes in Cl- transporter activity. Neuron. 2003 Aug 28; 39(5): 807-20.

40. Massimini M, Huber R, Ferrarelli F, Hill S, Tononi G. The Sleep Slow Oscillation as a Traveling Wave, The Journal of Neuroscience 2004;24: 6862-6870.

41. Euston D, Tatsuno M, McNaughton B. Fast-Forward Playback of Recent Memory Sequences in Prefrontal Cortex During Sleep, Science 2007;318, 1147.

42. Mak-McCully RA, Deiss SR, Rosen BQ, Jung KY, Sejnowski TJ, Bastuji H, Rey M, Cash S, Bazhenov M, Halgren, E. Synchronization of isolated downstates (K-complexes) may be caused by cortically-induced disruption of thalamic spindling. PLoS Comput Biol 2014;10(9), e1003855.

43. Tsai YT, Chan HL, Lee ST, Tu PH, Chang BL, Wu T. Significant thalamocortical coherence of sleep spindle, theta, delta, and slow oscillations in NREM sleep: Recordings from the human thalamus. Neuroscience Letters 2010;485: 173-177. doi:10.1016/j.neulet.2010.09.004.

44. Kaynak H, Altintaş A, Kaynak D, Uyanik Ö, Saip S, Ağaoğlu J, Önder G, Siva A. Fatigue and sleep disturbance in multiple sclerosis. European Journal of Neurology 2006;13(12), 1333-1339.

45. Steriade M. Sleep, epilepsy and thalamic reticular inhibitory neurons. Trends in neurosciences 2005;28(6), 317-324. 
Publication acceptée par Brain Injury (identifiant TBIN-2016-0272) à paraître en 2017

46. Fuentealba P, Steriade M. The reticular nucleus revisited: intrinsic and network properties of a thalamic pacemaker. Progress in neurobiology 2005;75(2), 125-141.

47. Tartaglia MC, Narayanan S, Francis SJ, Santos AC, De Stefano N, Lapierre Y, Arnold DL. The relationship between diffuse axonal damage and fatigue in multiple sclerosis. Archives of neurology 2004;61(2), 201-207

48. Touzet C. The Theory of Neural Cognition Applied to Robotics, International Journal of Advanced Robotic Systems 2015;12:74. doi: 10.5772/60693.

49. Castriotta RJ, Lai JM. Sleep disorders associated with traumatic brain injury. Arch Phys Med Rehabil 2001;82:1403-6.

50. Cohen M, Oksenberg A, Snir D, Stern MJ, Groswasser Z. Temporally related changes of sleep complaints in traumatic brain injured patients. J Neurol Neurosurg Psychiatry 1992;55:313-5.

51. Clinchot DM, Bogner J, Mysiw WJ, Fugate L, Corrigan J. Defining sleep disturbance after brain injury. Am J Phys Med Rehabil 1998;77:291-5.

52. Ziino C, Ponsford J. Measurement and prediction of subjective fatigue following traumatic brain injury. J Int Neuropsychol Soc 2005;11:416-25.

53. Braley TJ, Chervin RD. Fatigue in multiple sclerosis: mechanisms, evaluation, and treatment. Sleep 2010;33(8), 1061.

54. DeMarchi R, Bansal V, Hung A, Wroblewski K, Dua H, Sockalingam S, Bhalerao S. Review of awakening agents. Can J Neurol Sci 2005;32:4-17.

55. Marshall L, Helgadóttir H, Mölle M, Born J. Boosting slow oscillations during sleep potentiates memory. Nature 2006;444(7119), 610-613.

56. Ngo HVV, Miedema A, Faude I, Martinetz T, Mölle M, Born J. Driving Sleep Slow Oscillations by Auditory Closed-Loop Stimulation-A Self-Limiting Process. The Journal of Neuroscience 2015;35(17), 6630-6638.

57. Abeln V, Kleinert J, Strüder HK, Schneider S. Brainwave entrainment for better sleep and post-sleep state of young elite soccer players-A pilot study. European journal of sport science 2014;14(5), 393-402.

58. Raymann RJ, Swaab DF, Van Someren EJ. Skin deep: enhanced sleep depth by cutaneous temperature manipulation. Brain 2008;131(2), 500-513.

59. Plihal W, Born J. Effects of early and late nocturnal sleep on declarative and procedural memory. Journal of cognitive neuroscience 1997;9(4), 534-547.

60. Wiseman-Hakes C, Murray B, Moineddin R, Rochon E, Cullen N, Gargaro J, Colantonio A. Evaluating the impact of treatment for sleep/wake disorders on recovery of cognition and communication in adults with chronic TBI. Brain Inj. 2013;1364-1376

61. Touzet C. Morvan's syndrome and the sustained absence of all sleep rhythms for months or years: an hypothesis. Med. Hypotheses 2016;94:51-54, 2016. 